\title{
How to achieve long-term breast-feeding: factors associated with early discontinuation
}

\author{
Aysu Duyan Camurdan ${ }^{1, *}+$, Mustafa N Ilhan ${ }^{2}$, Ufuk Beyazova ${ }^{1}$, Figen Sahin ${ }^{1}$, \\ Nilgun Vatandas ${ }^{1}$ and Sancar Eminoglu ${ }^{3}$ \\ 'Department of Social Pediatrics, Gazi University School of Medicine, Ankara, Turkey: ${ }^{2}$ Department of Public \\ Health, Gazi University School of Medicine, Ankara, Turkey: ${ }^{3}$ Department of Pediatrics, Gazi University School \\ of Medicine, Ankara, Turkey
}

Submitted 28 May 2007: Accepted 24 November 2007: First published online 18 February 2008

\begin{abstract}
Objectives: To evaluate the factors associated with discontinuation of breastfeeding before 12 months in order to make suggestions for achieving long-term breast-feeding.

Design: A descriptive cross-sectional study.

Setting: Gazi University Medical School, Ankara, Turkey.

Subjects: Mothers of 1230 children who discontinued breast-feeding at least $15 \mathrm{~d}$ before the last visit were asked to fill out a questionnaire about the discontinuation process. Logistic regression analysis was performed to assess the independent effects of factors that might influence breast-feeding discontinuation. Results: Mean breast-feeding duration of the study group was 11.04 (SD 7.45) months. Introduction of bottle-feeding correlated with discontinuation of breast-feeding ( $r=0.507, P=0 \cdot 001$ ). Important risk factors for discontinuation of breast-feeding before the first 12 months were not exclusively breast-feeding at 3 and 6 months, prematurity, not having a plan about breast-feeding duration and maternity leave duration of $\leq 91 \mathrm{~d}$ for working mothers. The common reasons for abandoning breastfeeding in the first and second 6 months were similar, namely the mother's concerns about the sufficiency of breast milk. After 12 months and 18 months the reasons for discontinuation were the baby's unwillingness to eat solid foods while breast-feeding and the mother's perception that 'the baby is old enough', respectively.

Conclusion: The factors that improve long-term breast-feeding are successful exclusive breast-feeding in the first few months, intention of the mother to breastfeed and sufficient duration of maternity leave. This study emphasizes the importance of successful breast-feeding counselling during the first few months to achieve the desired long-term breast-feeding.
\end{abstract}

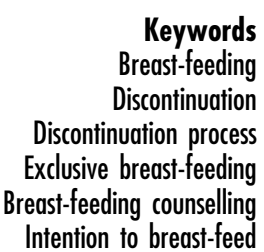

Keywords Discontinuation Discontinuation process Intention to breast-feed
Research documents numerous advantages for infants, mothers, families and society from breast-feeding and the use of human milk for infant feeding ${ }^{(1)}$. The WHO recommends that breast-feeding should preferably continue beyond the first year of life, and in populations with high rates of infection continued breast-feeding throughout the second year and longer is likely to benefit the infant ${ }^{(2)}$. Breast-feeding benefits are not only limited to the early months of the infant's life; breast-feeding beyond 12 months of age has been shown to have longterm protective effects from chronic diseases such as type 1 diabetes mellitus, coeliac disease, inflammatory bowel diseases and childhood cancers ${ }^{(3)}$.

†Correspondence address: Devlet mahallesi, Sapka Devrimi Caddesi, Yesiloz sitesi 2/5, Eryaman 06374, Ankara, Turkey.
It may be necessary for the breast-feeding pattern and its determinants to be recognized in each culture. Most of the studies on this subject have been performed in economically developed countries ${ }^{(4-9)}$ or very poor countries of Africa and $\mathrm{Asia}^{(10-14)}$. There is a limited number of studies from most of the developing world ${ }^{(15-18)}$ and only a few of them have originated from countries in the Eastern Mediterranean and Middle East region ${ }^{(19-21)}$. In Turkey, which is a developing Eastern Mediterranean country, there are only limited studies $^{(22-24)}$. In one, the factors that affect the duration of breast-feeding were investigated in a sample of 301 working mothers ${ }^{(22)}$. The other two studies had smaller sample sizes and shorter duration of follow-up ${ }^{(23,24)}$. In the present study we aimed to determine mean breast-feeding duration and evaluate the factors associated with discontinuation of breast-feeding before 12 months in a relatively larger sample 
of mothers of medium-high socio-economic status in a population where, culturally and religiously ${ }^{(25)}$, long-term breast-feeding beyond 12 months is accepted ${ }^{(26)}$ and women actively participate in occupational life. The reasons for and methods of discontinuation, and the effects of discontinuation on mothers and infants, were also investigated in detail as the mothers were followed until the end of their breast-feeding period in our clinic.

\section{Subject and methods}

\section{Subjects}

A descriptive cross-sectional study was performed at Gazi University Hospital, which was certified as a BabyFriendly Hospital in 2002. The well-child clinic in this hospital usually serves a population of medium-high socio-economic status and about 1400 newborns start follow-up visits each year. Infants are seen six times (at $15 \mathrm{~d}$, then 2, 4, 6, 9 and 12 months) in the first year of life; three times (at 15, 18 and 24 months) in the second year; and once annually thereafter until they are 5 years old. At each visit, their growth, development and feeding patterns are noted. The recommendation in this clinic about infant feeding includes exclusive breast-feeding in the first 6 months and continuing breast-feeding until 2 years of age with appropriate complementary foods. Exclusive breast-feeding (EBF) is defined as no other food or drink, including water or herbal teas, for 6 months of life ${ }^{(1)}$.

The infants and children between birth and 5 years of age who attended our well-child clinic between 1 January and 31 December 2004, and discontinued breast-feeding permanently at least $15 \mathrm{~d}$ before this visit, were included in the present study. They were not enrolled if they were continuing with breast-feeding or had not permanently discontinued in the last $15 \mathrm{~d}$. In other words, included infants were the ones who were breast-feeding in the previous visit and had discontinued breast-feeding thereafter; therefore they were enrolled if the visit was the first visit in the year that the infant had discontinued breastfeeding. This helped to avoid recurrent enrolment of the mother/child dyads who attended several times during the year of the study and also, as the time between two subsequent visits was usually not long, mothers could remember the details of discontinuation easily. Infants who were hospitalized in the neonatal intensive care unit or had congenital malformations, abnormalities or underlying diseases that could possibly alter the pattern of feeding in infancy were excluded. The mothers of 1230 children who fulfilled the inclusion criteria filled out a questionnaire about their discontinuation process. None of the mothers who fulfilled the inclusion criteria refused to participate.

\section{Statistical methods}

The data were analysed using the Statistical Package for the Social Sciences (SPSS for Windows) statistical software package version 10.0 (SPSS Inc., Chicago, IL, USA). Continuous variables are presented as means with their standard deviation, categorical variables as percentages. In statistical analysis, the $t$ test, ANOVA, the $\chi^{2}$ test and logistic regression analysis were performed to assess the independent effects of these predictors on breast-feeding discontinuation. Statistical significance was considered at $P<0 \cdot 05$. For the binary logistic regression model, gestational age $\leq 37$ weeks, maternity leave less than the official time ( $\leq 91 \mathrm{~d}$; housewives were modelled as having maternity leave $>91 \mathrm{~d}$ ), Caesarean section as type of birth, pacifier use in the first month of life, no plans about breast-feeding intention of the mother and not exclusively breast-feeding at 3 and 6 months were defined as risk factors.

\section{Results}

\section{Demographic characteristics}

Mean birth weight of the infants was 3101 (SD 37) g. Mean age of the mothers was 30.56 (SD 5.03) years and mean maternity leave of working mothers was $169 \cdot 50$ (SD $117 \cdot 40) \mathrm{d}$, with a median of $135 \mathrm{~d}$. Of the infants, $572(46.5 \%)$ were the first child in the family while $658(53.5 \%)$ were second or more in birth order. Mean duration of breast-feeding was 11.09 (SD 7.63) months for the first children and $10 \cdot 58$ (SD $7 \cdot 18$ ) months for the second or later children of the family. This difference was not statistically significant $(P=0 \cdot 735)$.

\section{Duration of breast-feeding}

Mean duration of breast-feeding of the infants included in this study was 11.04 (SD 7.45) months (minimum $1 \mathrm{~d}$; maximum 36 months) with a median of 10 months. At 1 , 3, 6, 12, 18 and 24 months, 95·3\%, 86 $0 \%$, 65·7\%, 39·0 \% $14 \cdot 3 \%$ and $4.0 \%$ of the infants, respectively, were continuing breast-feeding. In the study period there were no mothers who breast-fed their children beyond 36 months. EBF rates at 1,3 and 6 months were $92 \cdot 9 \%, 81 \cdot 1 \%$ and $41.7 \%$, respectively. The factors associated with the duration of breast-feeding are given in Table 1 .

Although the mean duration of breast-feeding was shorter among the mothers who were employed $(P=0 \cdot 017)$, these mothers had a longer EBF period than the non-working mothers $(3.50$ (SD 1.22) months $v .2 .90$ (sD 1.59) months, respectively, $P=0 \cdot 008$ ). Mean maternity leave was correlated with both overall duration of breast-feeding and EBF duration $(r=0 \cdot 196$ and $0 \cdot 160$, respectively, $P=0 \cdot 001)$.

More than half of the babies (51.5\%) used pacifiers; the mean time to start a pacifier was $59 \cdot 45$ (SD 88.26) d with a median of $30 \mathrm{~d}$. The early use of a pacifier was common, $57 \cdot 4 \%$ starting in the first month. The starting time of pacifier use and discontinuation of breast-feeding were weakly correlated $(r=0 \cdot 096, P=0 \cdot 019)$. 
Table 1 Factors associated with the overall duration of breast-feeding: mothers of medium-high socio-economic status, Ankara, Turkey, 2004 (n 1230)

\begin{tabular}{|c|c|c|c|c|c|}
\hline \multirow[b]{2}{*}{ Factor } & \multicolumn{2}{|c|}{ Frequency } & \multicolumn{2}{|c|}{ Overall duration of breast-feeding (months) } & \multirow[b]{2}{*}{$P$} \\
\hline & $n$ & $\%$ & Mean & SD & \\
\hline \multicolumn{6}{|l|}{ Infant's gender } \\
\hline Female & 624 & $50 \cdot 7$ & $10 \cdot 95$ & $7 \cdot 17$ & \multirow{2}{*}{ NS } \\
\hline Male & 606 & $49 \cdot 3$ & $11 \cdot 16$ & $7 \cdot 74$ & \\
\hline \multicolumn{6}{|l|}{ Birth weight $(\mathrm{g})$} \\
\hline 2499 & 188 & $15 \cdot 3$ & $6 \cdot 18$ & $5 \cdot 04$ & \multirow[t]{3}{*}{$0 \cdot 001 \ddagger$} \\
\hline 2500-3499 & 712 & $57 \cdot 9$ & $10 \cdot 98$ & $7 \cdot 26$ & \\
\hline$\geq 3500$ & 330 & $26 \cdot 8$ & $12 \cdot 87$ & $7 \cdot 69$ & \\
\hline \multicolumn{6}{|l|}{ Gestational age (weeks) } \\
\hline$\leq 37$ & 420 & $34 \cdot 1$ & $6 \cdot 66$ & $5 \cdot 31$ & \multirow{2}{*}{$0 \cdot 001$} \\
\hline$>37$ & 810 & $65 \cdot 9$ & $12 \cdot 22$ & $7 \cdot 43$ & \\
\hline \multicolumn{6}{|l|}{ Type of birth } \\
\hline Normal vaginal delivery & 382 & $31 \cdot 1$ & $12 \cdot 99$ & $7 \cdot 88$ & \multirow{2}{*}{$0 \cdot 001$} \\
\hline Caesarean section & 848 & $68 \cdot 9$ & $9 \cdot 77$ & $6 \cdot 96$ & \\
\hline \multicolumn{6}{|l|}{ Pacifier use } \\
\hline Yes & 634 & $51 \cdot 5$ & $8 \cdot 57$ & $6 \cdot 11$ & \multirow[t]{2}{*}{$0 \cdot 001$} \\
\hline No & 596 & $48 \cdot 5$ & $13 \cdot 86$ & $7 \cdot 80$ & \\
\hline \multicolumn{6}{|l|}{ Bottle-feeding } \\
\hline Yes & 952 & $77 \cdot 3$ & $9 \cdot 25$ & $6 \cdot 38$ & \multirow[t]{2}{*}{$0 \cdot 001$} \\
\hline No & 277 & $22 \cdot 7$ & $17 \cdot 53$ & $7 \cdot 44$ & \\
\hline \multicolumn{6}{|l|}{ EBF in 3rd month } \\
\hline EBF in first 3 months & 1006 & $81 \cdot 1$ & $12 \cdot 31$ & $6 \cdot 95$ & \multirow[t]{2}{*}{$0 \cdot 001$} \\
\hline No EBF in first 3 months & 224 & $18 \cdot 9$ & $5 \cdot 03$ & $6 \cdot 99$ & \\
\hline \multicolumn{6}{|l|}{ EBF in 6th month } \\
\hline EBF in first 6 months & 513 & $41 \cdot 7$ & $14 \cdot 66$ & $6 \cdot 66$ & \multirow[t]{2}{*}{$0 \cdot 001$} \\
\hline No EBF in first 6 months & 717 & $58 \cdot 3$ & $8 \cdot 42$ & $6 \cdot 88$ & \\
\hline \multicolumn{6}{|l|}{ Mother's age (years) } \\
\hline$\leq 29$ & 524 & $42 \cdot 6$ & $10 \cdot 69$ & $6 \cdot 76$ & \multirow[t]{3}{*}{ NS } \\
\hline $30-34$ & 428 & $34 \cdot 8$ & $11 \cdot 19$ & $7 \cdot 75$ & \\
\hline$\geq 35$ & 278 & $22 \cdot 6$ & 11.51 & $8 \cdot 25$ & \\
\hline \multicolumn{6}{|l|}{ Maternal education (years) } \\
\hline Primary school (0-8) & 318 & $25 \cdot 9$ & $11 \cdot 39$ & $8 \cdot 17$ & \multirow[t]{3}{*}{ NS } \\
\hline High school (9-11) & 205 & $16 \cdot 7$ & $11 \cdot 14$ & $7 \cdot 58$ & \\
\hline University (>11) & 707 & $57 \cdot 4$ & $10 \cdot 99$ & $7 \cdot 47$ & \\
\hline Maternal occupational status & & & & & \\
\hline Employed & 704 & $57 \cdot 2$ & $10 \cdot 61$ & $7 \cdot 01$ & $0 \cdot 017$ \\
\hline Housewife & 526 & $42 \cdot 8$ & $11 \cdot 65$ & $7 \cdot 99$ & \\
\hline Duration of maternity leave (d) & & & & & \\
\hline$\leq 91$ & 256 & $36 \cdot 6$ & $9 \cdot 03$ & $6 \cdot 45$ & $0.001 \ddagger$ \\
\hline $91-180$ & 232 & $32 \cdot 8$ & $10 \cdot 56$ & $7 \cdot 13$ & \\
\hline$>181$ & 217 & $30 \cdot 6$ & $12 \cdot 40$ & $6 \cdot 83$ & \\
\hline Maternal plans about breast-fe & & & & & \\
\hline Planned to breast-feed & 852 & $69 \cdot 2$ & $11 \cdot 58$ & $7 \cdot 62$ & 0.001 \\
\hline Not planned to breast-feed & 378 & $30 \cdot 8$ & $9 \cdot 97$ & $6 \cdot 93$ & \\
\hline
\end{tabular}

EBF, exclusive breast-feeding.

tOfficial maternity leave with salary in Turkey is maximum $91 \mathrm{~d}$; unpaid leave until 6 months is optional.

$\ddagger$ All three groups are different.

The mean time for starting bottle-feeding was $134 \cdot 85$ (SD $151 \cdot 20$ ) $d$ with a median of $90 \mathrm{~d}$, and $77 \cdot 3 \%$ of the babies had used a bottle for feeding at some time during the first year. Introduction of bottle-feeding correlated with the discontinuation of breast-feeding $(r=0.507$, $P=0 \cdot 001$ ).

The mothers who had an intention about the duration of breast-feeding comprised $69 \cdot 2 \%$ of the total. The planned duration and actual duration were not exactly the same $(16 \cdot 21$ (SD 7.63) months $v$. 11.58 (SD 7.62) months, respectively, $P=0 \cdot 001)$, but they were correlated $(r=0 \cdot 476, P=0 \cdot 001)$.

Table 2 demonstrates the results of logistic regression analyses of the factors that affected discontinuation of breast-feeding before 12 months. Significant risk factors in the discontinuation of breast-feeding before 12 months were: not exclusively breast-feeding at 3 and 6 months, no intention about the duration of breast-feeding, gestational age $\leq 37$ weeks and maternity leave $\leq 91 \mathrm{~d}$.

\section{Motbers' perception about discontinuation of breast-feeding}

The mothers' answers about the reasons for abandoning breast-feeding before 6 months, at 6-12 and 13-18 months and after 18 months are given in Table 3 . The common reasons for abandoning breast-feeding in the first and second 6 months were similar, namely the 
Table 2 Significant risk factors in the discontinuation of breast-feeding before 12 months: mothers of medium-high socio-economic status, Ankara, Turkey, 2004 (n 1230)

\begin{tabular}{|c|c|c|}
\hline \multirow[b]{2}{*}{ Risk factor } & \multicolumn{2}{|c|}{ Relative risk $(95 \% \mathrm{Cl})$ for discontinuation of breast-feeding before 12 months } \\
\hline & EBF in 3rd month & EBF in 6th month \\
\hline \multicolumn{3}{|l|}{ EBF } \\
\hline Yes & 1.00 (referent) & 1.00 (referent) \\
\hline No & $6 \cdot 10(2 \cdot 36,15 \cdot 80)^{\star}$ & $4 \cdot 14(2 \cdot 55,6 \cdot 70)^{\star}$ \\
\hline \multicolumn{3}{|c|}{ Maternal intention to breast-feed } \\
\hline Yes & $1 \cdot 00$ (referent) & 1.00 (referent) \\
\hline No & $2 \cdot 27(1 \cdot 34,3 \cdot 83)^{\star}$ & $2 \cdot 14(1 \cdot 26,3 \cdot 63)^{\star}$ \\
\hline \multicolumn{3}{|l|}{ Gestational age (weeks) } \\
\hline$>37$ & 1.00 (referent) & 1.00 (referent) \\
\hline$\leq 37$ & $3.03(1 \cdot 72,5 \cdot 34)^{*}$ & $2 \cdot 30(1 \cdot 32,4 \cdot 01)^{*}$ \\
\hline \multicolumn{3}{|c|}{ Duration of maternity leave (d) } \\
\hline$>91$ & 1.00 (referent) & 1.00 (referent) \\
\hline$\leq 91$ & $2 \cdot 19(1 \cdot 11,4 \cdot 30)^{\star}$ & $1 \cdot 78(1 \cdot 15,3 \cdot 41)^{\star}$ \\
\hline \multicolumn{3}{|l|}{ Type of birth } \\
\hline Vaginal delivery & 1.00 (referent) & $1 \cdot 00$ (referent) \\
\hline Caesarean section & $1 \cdot 47(0 \cdot 87,2 \cdot 47)$ & $1 \cdot 39(0 \cdot 83,2 \cdot 35)$ \\
\hline \multicolumn{3}{|l|}{ Pacifier in 1st month } \\
\hline No & 1.00 (referent) & 1.00 (referent) \\
\hline Yes & $0.97(0.60,1.56)$ & $1.08(0.67,1 \cdot 75)$ \\
\hline
\end{tabular}

EBF, exclusive breast-feeding.

Factor was significantly associated with breast-feeding discontinuation: ${ }^{\star} P<0.05$.

Table 3 Mothers' perception/motives to discontinue breast-feeding according to termination time: mothers of medium-high socio-economic status, Ankara, Turkey, 2004 ( $n$ 1230)

\begin{tabular}{|c|c|c|c|c|c|c|c|c|}
\hline & \multicolumn{8}{|c|}{ Termination time } \\
\hline & \multicolumn{2}{|c|}{$<6$ months } & \multicolumn{2}{|c|}{$6-12$ months } & \multicolumn{2}{|c|}{ 13-18 months } & \multicolumn{2}{|c|}{$>18$ months } \\
\hline & Mean & SD & Mean & SD & Mean & SD & Mean & SD \\
\hline \multirow[t]{2}{*}{ Discontinuation time of breast-feeding (months) } & $3 \cdot 02$ & $1 \cdot 58$ & $8 \cdot 71$ & $2 \cdot 21$ & $16 \cdot 01$ & $1 \cdot 88$ & $24 \cdot 43$ & $5 \cdot 74$ \\
\hline & $n$ & $\%$ & $n$ & $\%$ & $n$ & $\%$ & $n$ & $\%$ \\
\hline \multicolumn{9}{|c|}{ Reasons given by mothers for breast-feeding discontinuation } \\
\hline Insufficient breast milk & 180 & $54 \cdot 4$ & 227 & $52 \cdot 3$ & 56 & $18 \cdot 3$ & 10 & $6 \cdot 5$ \\
\hline Introduction of bottle & 53 & $15 \cdot 9$ & 37 & $8 \cdot 5$ & 5 & $1 \cdot 7$ & 1 & $0 \cdot 7$ \\
\hline 'Baby is old enough' & - & 0 & 17 & $3 \cdot 8$ & 58 & $19 \cdot 0$ & 86 & $54 \cdot 3$ \\
\hline Subjective reasons related to mothert & 25 & $7 \cdot 4$ & 54 & $12 \cdot 7$ & 46 & $14 \cdot 7$ & 18 & $11 \cdot 2$ \\
\hline Mother’s illnessł & 37 & $11 \cdot 2$ & 47 & $10 \cdot 7$ & 44 & $14 \cdot 4$ & 12 & $7 \cdot 5$ \\
\hline Baby's refusal of other solid foods & 3 & $1 \cdot 1$ & 17 & $3 \cdot 8$ & 78 & $25 \cdot 7$ & 23 & $14 \cdot 5$ \\
\hline New pregnancy of the mother & 6 & $1 \cdot 8$ & 20 & $4 \cdot 5$ & 17 & $5 \cdot 3$ & 8 & $5 \cdot 3$ \\
\hline Acute health problem of the child & 27 & $8 \cdot 0$ & 16 & $3 \cdot 7$ & 2 & 0.6 & - & 0 \\
\hline Total $(n 1230)$ & 331 & $27 \cdot 0$ & 435 & $35 \cdot 3$ & 306 & $24 \cdot 6$ & 158 & $12 \cdot 9$ \\
\hline
\end{tabular}

tFrequent awakening at night, the thought of 'It will be harder if I discontinue later', willingness to fast during Ramadan, to be tired of breast-feeding, willingness to diet to lose weight.

‡Physical or mental illness of the mother that necessitates drug use (postpartum depression, rheumatoid arthritis, migraine), nipple problems (sore or retracted), breast problems (engorgement, mastitis).

mother's concerns about the sufficiency of breast milk. The mothers who discontinued breast-feeding due to perceived milk insufficiency started complementary foods earlier (4.26 (SD 1.91) months v. 4.59 (SD 2.12) months, respectively, $P=0 \cdot 010)$ and used a pacifier and bottle more frequently than others (pacifier $72 \cdot 0 \% \mathrm{v}$. $43.4 \%$, respectively; bottle $85.9 \% v .74 \cdot 2 \%$, respectively, $P=0 \cdot 001)$. The most common reason for discontinuation was the baby's unwillingness to eat solid foods in 12-month-old infants, while for 18-month-old infants it was the mother's perception that 'the baby is old enough'.
Less than half of the mothers ( $n 577,46.9 \%$ ) stated that although they wanted to continue breast-feeding they could not succeed, while the other half ( $n$ 653, 53.1\%) said that they themselves decided to stop breast-feeding. Of the mothers who decided to discontinue, $65.6 \%$ ( $n$ 428) reported that they used a method. Methods that were used for discontinuation were as follows:

- Saying 'There is no milk anymore', 'The breast is ill', etc. $(n 176,41 \cdot 1 \%)$.

- Using something that changes the appearance and odour of the breast ( $n$ 110, 25.7\%). 
- Gradual discontinuation (first discontinuation in the day time, then at night time) ( $n 88,20 \cdot 5 \%)$.

- Not being home for a few days due to work-related journeys ( $n$ 54, 12.6\%).

In the cases where breast-feeding was discontinued after 18 months ( $n$ 158), the method of putting on something that changed the appearance and odour of the breast was the most popular $(46 \cdot 2 \%)$. Of the seventythree mothers who used such a method with the aim of making the baby disgusted with the breast, nineteen used tomato sauce, twelve used molasses, nine used cosmetics such as nail polish and lipstick, eight covered the nipple with tape and six with dye, while five attached black hair or wool to the nipple. Some mothers also tried putting salty lemon juice or merely salt $(n 4)$, red pepper $(n 3)$, cocoa or coffee $(n 3)$, cosmetic cream $(n 2)$, charcoal ( $n$ 1) or garlic $(n 1)$ on the nipple to change the taste and odour.

\section{Discussion}

\section{Duration of breast-feeding}

There are only a few studies that specifically evaluate long-term breast-feeding patterns and associated factors $^{(7,10,19)}$. In the current study, breast-feeding patterns and associated factors of a relatively large sample (1230 subjects) who continued breast-feeding for as long as 36 months were evaluated.

Although the ideal EBF duration is defined as 6 months $^{(1)}$, studies performed in both developed and developing countries demonstrate that this goal is currently not achieved $^{(4,5,15,16,27)}$. In Norway, where breast-feeding rates are high, a recent study revealed that the EBF rate in the first month was $90 \%$, falling rapidly to $7 \%$ at 6 months $^{(5)}$. In the present study these figures were $92.9 \%$ and $41.7 \%$, respectively. We tried to determine EBF duration that affects overall breast-feeding duration, and we saw that mothers who breast-fed exclusively in the first 3 months were six times more likely to continue breastfeeding until 12 months. Some other studies also report that early introduction of formula or milk is associated with shorter breast-feeding duration ${ }^{(4,7,15-17)}$, but the critical importance of EBF duration for overall breast-feeding of at least 12 months is emphasized in the present study.

In our study group none of the infants had stayed in the neonatal intensive care unit. All of the premature infants were healthy and were discharged directly from the maternity ward, but still their duration of breast-feeding was shorter than that of term infants. The infants whose gestational age was $\leq 37$ weeks were two or three times more likely to discontinue breast-feeding before 12 months. Low birth weight of premature infants might have contributed to this result as well. Although there are studies that report no significant association between birth weight and breast-feeding duration ${ }^{(28,29)}$, the present study together with some others reveal that low birth weight has a negative impact on duration of breast-feeding ${ }^{(5,8,20,30)}$.

In recent years, in addition to demographic factors, maternal intention about the infant's feeding has been shown to be a very strong indicator of breast-feeding duration $^{(7,16,19,23,31,32)}$. Asking women about their plans for the duration of breast-feeding is a useful way to predict the risk of short breast-feeding duration. In the present study, mothers who had a plan about breastfeeding duration continued longer than other mothers. These results confirm the findings of previous studies in which breast-feeding duration goal is found to be an important predictor of longer breast-feeding.

It is reported that mothers who return to full-time work in the first year have a shorter duration of breastfeeding ${ }^{(19)}$. In Turkey paid leave is $91 \mathrm{~d}$ maximally and mothers can get unpaid leave up to 6 months. The mothers in the present study had an average maternity leave duration of $169 \mathrm{~d}$ and mean breast-feeding duration was approximately 1 month shorter for employed mothers compared with housewives, but EBF period was longer. EBF was correlated with the length of maternity leave. This may not be interpreted as the negative effect of employment on breast-feeding, but it may rather be due to the mother's decision to postpone working until the baby stops breast-feeding or starts complementary foods. As our study demonstrates that successful EBF in the first 3 months increases the likelihood of continuing breast-feeding until 12 months, if breast-feeding is supported in the workplace by breast pumps and appropriate storage conditions for breast milk, mothers can start working after 3 months to minimize economic loss.

Multiparous women have been shown to continue breast-feeding longer than first-time mothers ${ }^{(19,33)}$. This is thought to be due to breast-feeding experience of the mother after the first child, but there are also other studies which report no difference ${ }^{(7,10,16)}$. The present study did not demonstrate any difference between duration of breast-feeding of first and second children of the family.

In the present study mean duration of breast-feeding was shorter in Caesarean deliveries, but multivariate analysis did not reveal a significant effect of type of birth. Other studies report that type of birth may be associated $^{(33-35)}$, may not be associated ${ }^{(6,7,23)}$ or may be weakly associated ${ }^{(36)}$ with breast-feeding duration.

There is controversy about the effects of pacifiers on breast-feeding duration. Many studies report a strong negative association between pacifier use in the first month and breast-feeding duration ${ }^{(7,8,16,37,38)}$ while others find a weaker association $^{(39,40)}$ and one study reports that pacifier use does not increase the risk of early weaning per $\mathrm{se}^{(41)}$. In the present study mean breast-feeding duration was shorter in pacifier users and there was a weak correlation between pacifier use and termination of breast-feeding.

In many studies it is demonstrated that breast-feeding duration is shorter if the infant becomes used to 
bottle-feeding $^{(7,20,37-40)}$. The present study supports this finding. The reason to start bottle-feeding is usually the mother's inappropriate belief about her insufficient milk supply, which ends up with the baby's confusion about the sucking technique and diminution of the mother's milk $^{(37)}$. It is not clear whether bottle-feeding is a reason or a result of insufficient milk supply ${ }^{(42,43)}$.

\section{Reasons for and processes of discontinuation of breast-feeding}

In the present study the most common reason stated by mothers for discontinuation of breast-feeding in the first 12 months was perception of 'insufficient breast milk'. Concerns about insufficient breast milk are universal and studies performed in different countries demonstrate that this concern has a negative effect on breast-feeding duration because of early supplementation ${ }^{(10,15,19)}$. In the present study the mothers who discontinued breastfeeding due to milk insufficiency had started complementary foods earlier, and had used a pacifier and bottle more frequently than the others. These findings suggest that the feeling of insufficient milk is a strong concern among mothers and results in them trying an alternative feeding method. This results in involuntary early discontinuation of breast-feeding.

After 12 months, the most common reason for discontinuation according to mothers was refusal of the infant to eat solid foods ${ }^{(44)}$. Most of the mothers in the present study believed the baby is old enough to terminate breast-feeding' when the baby was 12 months old. In some African countries discontinuation before 15-18 months is considered 'early',(10,13). However, in Germany and New Zealand, breast-feeding for $4-6$ months is considered as a 'long enough' duration by most families ${ }^{(4,7)}$. This emphasizes that discontinuation time due to the same reason may be very different in different societies.

When mothers decide to terminate because the infant is old enough, they choose a method according to their customs, traditions and beliefs. In Senegal 'abrupt' cessation is the method of choice and a healer performs a ritual on the day of weaning ${ }^{(13)}$. In the present study the most common methods were abrupt cessation and, especially after 18 months, use of something that changes the appearance and odour of the breast. In this period, babies have difficulty relinquishing the breast and mothers choose to act as if there is something wrong with it.

A limitation of the present study is that our report on duration of breast-feeding refers only to mother/child dyads who attended our clinic and had discontinued breast-feeding permanently at least $15 \mathrm{~d}$ before the visit. For this reason the reported duration does not refer to the general population. Also, some other factors that had been investigated in previous studies, such as family size and structure, maternal smoking status and BMI, were not investigated in the present study as its scope was already too wide.

\section{Conclusion}

The factors that affect long-term breast-feeding and the reasons why mothers choose to terminate breast-feeding are important items of information for a health-care worker. The present study emphasizes the importance of successful breast-feeding counselling during the first few months to achieve the desired long-term breast-feeding.

\section{Acknowledgements}

Contributors: A.D.C. had primary responsibility for protocol development, patient screening, enrolment, outcome assessment, preliminary data analysis and writing the manuscript. M.N.I. and U.B. supervised the design and execution of the study, performed the final analyses and contributed to the writing of the manuscript. F.S. participated in the development of the protocol and analytic framework for the study and contributed to the writing of the manuscript. N.V. and S.E. were responsible for patient screening and contributed to the writing of the manuscript.

Source of funding: None.

Conflict of interest: There are no competing interests.

\section{References}

1. American Academy of Pediatrics Section on Breastfeeding (2005) Breastfeeding and the use of human milk. Pediatrics 115, 496-506.

2. World Health Organization (2003) Global Strategy for Infant and Young Child Feeding. http://www.who.int/ child-adolescent-ealth/New_Publications/NUTRITION/gs_ iycf.pdf

3. Piovanetti Y (2001) Breastfeeding beyond 12 months. Pediatr Clin North Am 48, 199-206.

4. Dulon M, Kersting M \& Schach S (2001) Duration of breastfeeding and associated factors in Western and Eastern Germany. Acta Paediatr 90, 931-935.

5. Lande B, Andersen LF, Baerug A, Trygg KU, Lund-Larsen K, Veierod MB \& Bjorneboe GE (2003) Infant feeding practices and associated factors in the first six months of life: the Norwegian infant nutrition survey. Acta Paediatr 92, 152-161.

6. Riva E, Banderali G, Agostoni C, Silano M, Radaelli G \& Giovannini M (1999) Factors associated with initiation and duration of breastfeeding in Italy. Acta Paediatr $\mathbf{8 8}$, 411-415.

7. Vogel A, Hutchison BL \& Mitchell EA (1999) Factors associated with the duration of breastfeeding. Acta Paediatr 88, 1320-1326.

8. Clements MS, Mitchell EA, Wright SP, Esmail A, Jones DR \& Ford RP (1997) Influences on breastfeeding in southeast England. Acta Paediatr 86, 51-56.

9. Bouvier P \& Rougemont A (1998) Breast-feeding in Geneva: prevalence, duration and determinants. Soz Praventivmed 43, 116-123.

10. Jakobsen MS, Sodemann M, Molbak K \& Aaby P (1996) Reason for termination of breastfeeding and the length of breastfeeding. Int J Epidemiol 25, 115-121.

11. Jakobsen MS, Sodemann M, Molbak K, Alvarenga IJ, Nielsen J \& Aaby P (2003) Termination of breastfeeding after 12 months of age due to a new pregnancy and other 
causes is associated with increased mortality in GuineaBissau. Int J Epidemiol 32, 92-96.

12. Simondon KB, Costes R, Delaunay V, Diallo A \& Simondon F (2001) Children's height, health and appetite influence mothers' weaning decisions in rural Senegal. Int $J$ Epidemiol 30, 476-481.

13. Mane NB, Simondon KB, Diallo A, Marra AM \& Simondon F (2006) Early breastfeeding cessation in rural Senegal: causes, modes, and consequences. Am J Public Health 96, 139-144.

14. Vaahtera M, Kulmala T, Hietanen A, Ndekha M, Cullinan T, Salin ML \& Ashorn P (2001) Breastfeeding and complementary feeding practices in rural Malawi. Acta Paediatr 90, 328-332.

15. Simic T, Sumanovic-Glamuzina D, Boranic M, Vuksic I \& Boban A (2004) Breastfeeding practices in Mostar, Bosnia and Herzegovina: cross-sectional self-report study. Croat Med J 45, 38-43.

16. Marques NM, Lira PI, Lima MC, da Silva NL, Filho MB, Huttly SR \& Ashworth A (2001) Breastfeeding and early weaning practices in northeast Brazil: a longitudinal study. Pediatrics 108, E66.

17. Berra S, Sabulsky J, Rajmil L, Passamonte R, Pronsato J \& Butinof M (2003) Correlates of breastfeeding duration in an urban cohort from Argentina. Acta Paediatr 92, 952-957.

18. England L, Brenner R, Bhaskar B, Simons-Morton B, Das A, Revenis M, Mehta N \& Clemens J (2003) Breastfeeding practices in a cohort of inner-city women: the role of contraindications. BMC Public Health 3, 1-9.

19. Hajian-Tilaki KO (2005) Factors associated with the pattern of breastfeeding in the north of Iran. Ann Hum Biol 32, 702-713.

20. Shiva F \& Nasiri M (2003) A study of feeding patterns in young infants. J Trop Pediatr 49, 89-92.

21. al-Ayed IH \& Qureshi MI (1998) Breastfeeding practices in urban Riyadh. J Trop Pediatr 44, 113-117.

22. Yilmaz G, Gurakan B, Akgun S \& Ozbek N (2002) Factors influencing breastfeeding for working mothers. Turk $J$ Pediatr 44, 30-34.

23. Alikasifoglu M, Erginoz E, Gur ET, Baltas Z, Beker B \& Arvas A (2001) Factors influencing the duration of exclusive breastfeeding in a group of Turkish women. J Hum Lact 17, 220-226.

24. Ertem IO, Akıncı Z, Ulukol B \& Baykan Gulnar S (2001) Socioeconomically advantaged infants attending a university well-child clinic in Ankara: are they breast-feeding optimally? Turk J Pediatr 43, 223-230.

25. Gatrad AR \& Sheikh A (2001) Muslim birth customs. Arch Dis Child Fetal Neonatal Ed 84, 6-8.

26. Institute of Population Studies (2004) Infant feeding practices and children's and women's nutritional status. Turkey Demographic and Health Survey TDHS - 2003. http://www.hips.hacettepe.edu.tr/tnsa2003eng/index.htm

27. Britton C, McCormick FM, Renfrew MJ, Wade A \& King SE (2007) Support for breastfeeding mothers. Cochrane Database Syst Rev 24, CD001141.
28. Scott JA, Aitkin I, Binns CW \& Aroni RA (1999) Factors associated with the duration of breastfeeding amongst women in Perth, Australia. Acta Paediatr 88, 416-421.

29. Mascarenhas MLW, Albernaz EP, Silva MB \& da Silveira RB (2006) Prevalence of exclusive breastfeeding and its determiners in the first 3 months of life in the South of Brazil. J Pediatr (Rio J) 82, 289-294.

30. Bicalco-Mancini PG \& Melendez GV (2004) Exclusive breast feeding at the point of discharge of high-risk newborns at a Neonatal Intensive Care Unit and the factors associated with this practice. J Pediatr (Rio J) 80, 241-248.

31. Ertem IO, Votto N \& Leventhal JM (2001) The timing and predictors of the early termination of breastfeeding. Pediatrics 107, 543-548.

32. Donath SM \& Amir LH (2003) Relationship between prenatal infant feeding intention and initiation and duration of breastfeeding: a cohort study. Acta Paediatr 92, 352-356.

33. Antoniou $E$, Daglas $M$, Iatrakis $G$, Kourounis $G$ \& Greatsas G (2005) Factors associated with initiation and duration of breastfeeding in Greece. Clin Exp Obstet Gynecol 32, 37-40.

34. Cattaneo A \& Buzzetti R (2001) Effect on rates of breast feeding of training for the Baby Friendly Hospital Initiative. BMJ 323, 1358-1362.

35. Leung GM, Ho LM \& Lam TH (2002) Breastfeeding rates in Hong Kong: a comparison of the 1987 and 1997 Birth Cohorts. Birth 29, 162-168.

36. Berra S, Rajmil L, Passamonte R, Fernandez E \& Sabulsky J (2001) Premature cessation of breastfeeding in infants: development and evaluation of a predictive model in two Argentinian cohorts: the CLACYD study, 1993-1999. Acta Paediatr 90, 544-551.

37. Righard L (1998) Are breastfeeding problems related to incorrect breastfeeding technique and the use of pacifiers and bottles? Birth 25, 40-44.

38. Cunha AJ, Leite AM \& Machado MM (2005) Breastfeeding and pacifier use in Brazil. Indian J Pediatr 72, 209-212.

39. Aarts C, Hornell A, Kylberg E, Hofvander Y \& GebreMedhin M (1999) Breastfeeding patterns in relation to thumb sucking and pacifier use. Pediatrics 104, e50.

40. Howard CR, Howard FM, Lanphear B, de Blieck EA, Eberly S \& Lawrence RA (1999) The effects of early pacifier use on breastfeeding duration. Pediatrics 103, E33.

41. Kramer MS, Barr RG, Dagenais S, Yang H, Jones P, Ciofani L \& Jane F (2001) Pacifier use, early weaning, and cry/fuss behavior: a randomized controlled trial. JAMA 286, 322-326.

42. Benis MM (2002) Are pacifiers associated with early weaning from breastfeeding? Adv Neonatal Care 2, 259-266.

43. Ullah S \& Griffiths P (2003) Does the use of pacifiers shorten breastfeeding duration in infants? Br J Community Nurs 8, 458-463.

44. Fawzi WW, Herrera MG, Nestel P, el Amin A \& Mohamed KA (1998) A longitudinal study of prolonged breastfeeding in relation to child undernutrition. Int J Epidemiol 27, 255-260. 